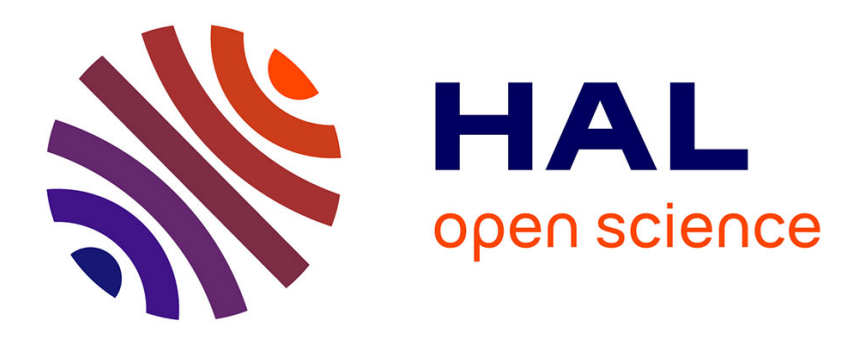

\title{
La normalisation du rôle parental par une équipe soignante.
}

Thomas Bonnet

\section{To cite this version:}

Thomas Bonnet. La normalisation du rôle parental par une équipe soignante.. Recherches familiales, 2015. hal-01898925

\section{HAL Id: hal-01898925 \\ https://hal.science/hal-01898925}

Submitted on 19 Oct 2018

HAL is a multi-disciplinary open access archive for the deposit and dissemination of scientific research documents, whether they are published or not. The documents may come from teaching and research institutions in France or abroad, or from public or private research centers.
L'archive ouverte pluridisciplinaire HAL, est destinée au dépôt et à la diffusion de documents scientifiques de niveau recherche, publiés ou non, émanant des établissements d'enseignement et de recherche français ou étrangers, des laboratoires publics ou privés. 


\section{La normalisation du rôle parental par une équipe soignante.}

\section{Thomas Bonnet ${ }^{1}$.}

\section{Résumé.}

L'étude des interactions entre une équipe soignante et les parents des patients hospitalisés dans un service pédiatrique montre un processus de normalisation du rôle parental. La catégorisation sociale des parents réalisée par les soignants fait ressortir l'image d'un modèle du " bon " parent. L'évaluation par les soignants de la distance à ce modèle permet d'envisager les actions à mettre en œuvre sur le parent pour le conformer à l'idéal professionnel. Ce dernier s'incarne dans une prise en charge globale de l'enfant, et implique donc une place particulière du parent.

\section{Introduction.}

L'hôpital s'organise selon un ordre social négocié à la fois temporaire et local structuré par les différentes logiques d'actions se rencontrant en son sein ${ }^{2}$. Sans aborder la question du travail d'articulation qui s'opère entre les différents segments professionnels, deux logiques importantes se rencontrent: la logique professionnelle des soignants et la logique profane des patients ${ }^{3}$. L'étude sociologique d'un service pédiatrique hospitalier révèle une relation triadique entre le soignant, le soigné et le parent. Le parent joue un rôle dans l'ordre social qui régit le fonctionnement du service, notamment lorsqu'il revendique ses compétences à s'occuper de son enfant. II est de surcroit encouragé par le développement du droit des usagers à participer à la co-construction des soins. L'équipe soignante est donc amenée à négocier sa logique professionnelle de mise en œuvre des soins avec la logique profane des parents. Autrement dit, la rencontre de ces deux logiques d'action, de ces deux régulations, imposent pour coopérer et dépasser l'antagonisme du rapport de force, une négociation et l'élaboration d'une "régulation conjointe ${ }^{4}$. La régulation conjointe est le résultat, selon la théorie de la régulation sociale, d'un accord entre une régulation de contrôle, en l'occurrence celle des soignants, et une régulation autonome, celle des parents. La régulation conjointe permet la coopération car elle vise l'élaboration de règles communes qui encadrent les échanges entre acteurs ${ }^{5}$. Mais la négociation des soins $n^{\prime}$ est pas toujours aisée. Certaines négociations aboutissent effectivement à la mise en place de règles du jeu

\footnotetext{
${ }^{1}$ Doctorant en sociologie, CERTOP, Université de Toulouse Jean Jaurès - tomhb@laposte.net.

${ }^{2}$ Anselm STRAUSS, La trame de la négociation, Paris, L'Harmattan, 1992.

${ }^{3}$ Isabelle BASZANGER, "Les maladies chroniques et leur ordre négocié ", Revue française de sociologie, vol 27, $\mathrm{n}^{\circ} 1, \mathrm{p}$ 3-27, 1986.

4 Jean-Daniel REYNAUD, "Les régulations dans les organisations: régulation de contrôle et régulation autonome ", Revue française de sociologie, vol 29, n¹, p 5-18, 1988.

${ }^{5}$ Gilbert de TERSSAC (dir), La théorie de la régulation sociale de Jean-Daniel Reynaud. Débats et prolongements, Paris, La Découvert, 2003.
} 
qui harmonisent les logiques en présence alors que d'autres compromis sont beaucoup plus précaires. II convient de souligner quelques aspects importants. Si l'équipe soignante s'engage dans des négociations autour du soin avec les parents, ce n'est pas seulement parce que depuis 1983, avec l'ouverture des services pédiatriques aux parents, la législation les y contraint ${ }^{6}$, c'est aussi parce que la participation du parent au soin relève de leur idéal professionnel. De plus, La participation parentale est un moyen pour l'équipe soignante de se constituer un confort de travail, dans le sens où un parent actif qui a intégré le rôle proposé par l'équipe soignante permet d'alléger le travail de cette dernière. Nous proposons donc dans cet article de nous placer du point de vue de l'équipe soignante et d'étudier le travail réalisé sur les parents des enfants hospitalisés pour les faire tendre vers leur idéal professionnel : en faire de "bons " parents. Nous interrogerons la place envisagée pour les parents par les soignants et les actions qu'ils mettent en œuvre pour les socialiser à ce rôle. En étudiant les pratiques et les représentations des soignants, nous verrons comment ils essaient de conformer les parents à cet idéal professionnel.

\section{Méthodologie et terrain d'enquête.}

Cet article s'appuie sur une immersion de trois mois et plus de 350 heures d'observation au sein d'une équipe soignante d'un service pédiatrique hospitalier. Ce travail ethnographique mené en blouse blanche nous a permis d'accompagner les professionnels dans les chambres des patients, nous fondre parmi l'équipe et observer les interactions entre les soignants et les usagers ${ }^{7}$, ainsi que les transmissions de soins lors des relèves entre équipe entrante et équipe sortante. Bien intégré au sein de l'équipe, malgré un biais de genre évident (nous étions un des seuls garçons du service), les enquêtés ont paru percevoir favorablement notre démarche. Les observations sont complétées par les résultats d'une campagne d'entretiens (26 entretiens individuels et collectifs d'une durée moyenne d'une demi-heure ont été menés auprès d'infirmières, d'auxiliaires et de la cadre du service). Les entretiens se sont déroulés à l'hôpital, lors des moments de pauses ou d' "accalmies", lorsque les acteurs étaient disponibles. Les observations en service de nuit ont constitué un moment favorable pour réaliser les plus longs entretiens.

La recherche s'est déroulée dans un $\mathrm{CHU}$ au sein d'un service pédiatrique qui regroupe quatre spécialités médicales: la néphrologie, l'endocrinologie, la neurologie et la cardiologie. II y a donc toute une palette de cas médicaux différents. On retrouve des patients " chroniques " comme les épileptiques ou les diabétiques qui fréquentent le service

\footnotetext{
${ }^{6}$ Sarra MOUGEL, Au chevet de l'enfant malade. Parents/professionnels, un modèle de partenariat ?, Paris, Armand Colin, 2009

${ }^{7}$ Le terme d' " usagers » nous permet de faire l'économie du couple enfant-parent mais c'est bien dans ce sens qu'il faut le comprendre. Effectivement, le parent est "omniprésent " dans les interactions soignant-soigné, puisqu'en fonction de l'âge du patient, le parent est éventuellement l'unique interlocuteur au sujet des soins. De fait, plus le patient est jeune plus le soignant s'adresse au parent.
} 
ponctuellement et deviennent par là même des " habitués » et des enfants hospitalisés pour une pathologie aiguë qui ne sont en principe admis que pour une unique fois. II y a des enfants hospitalisés pour des pathologies relativement bénignes et des enfants atteints de tumeur qui, pour certains d'entre eux, décéderont au sein du service. La durée d'hospitalisation des patients peut donc varier de quelques jours à plusieurs mois.

Le service dispose de 27 lits répartis dans 21 chambres. Celles-ci sont distribuées en quatre secteurs, non fondés sur les différentes pathologies, mais plutôt sur le partage du service en quatre binômes soignants, composé d'une infirmière et d'une auxiliaire de puériculture. L'équipe soignante dans son intégralité regroupe 17 infirmières et puéricultrices ainsi que 15 auxiliaires de puériculture. Une cadre de proximité assure le fonctionnement du service. L'équipe médicale est présente essentiellement en matinée, lors des visites médicales. L'effectif du service est à $98 \%$ féminin et la moyenne d'âge est de 35 ans.

La présence continue d'un parent est encouragée dans le service afin d'avoir des enfants, comme l'exprime la cadre, "moins angoissés, ce qui facilite les soins ". C'est la présence d'un unique parent qui est autorisée en continu et non le couple parental pour ne pas surcharger le service. Nous parlons donc du parent au singulier et " au féminin " car s'il est vrai que les soignants sont confrontés à une alternance du père et de la mère qui se relaient au chevet de l'enfant, ce sont plus souvent les mères qui sont présentes.

\section{Les soignantes et le parent.}

Intéressons-nous aux protagonistes principaux de l'article, à savoir les soignants et le parent présent. L'évolution du rôle infirmier a enrichi les actes techniques du soin incombant aux soignants tout en préservant les aspects relationnels. Ces derniers prennent une place importante étant donné que la place de l'usager a évolué également passant d'un usager plutôt passif et soumis au savoir médical à un usager plus actif et décisionnaire dans le processus de soin ${ }^{8}$. Ainsi, l'aspect relationnel du soin est mis en avant par les soignants pour permettre la négociation du soin et la prise en compte des logiques parentales.

\subsection{Les soignants.}

Le binôme soignant est composé d'une infirmière ou puéricultrice et d'une auxiliaire de puériculture. Le fonctionnement en binôme implique qu'elles réalisent les tours de soins ensemble, mais il faut néanmoins différencier les activités de l'infirmière et de l'auxiliaire. L'auxiliaire travaille sous la responsabilité de l'infirmière dans le cadre du rôle propre infirmier.

\footnotetext{
${ }^{8}$ François-Xavier SCHWEYER, «Le soin négocié, la participation des usagers et l'action publique ", in Simone PENNEC, Françoise LE BORGNE-UGUEN, Florence DOUGUET, (dir), Les négociations du soin, Rennes, Presses Universitaires de Rennes, p 27-40, 2014.
} 
En 1978, la loi du 31 mai, (78-615), a redéfini la pratique infirmière en insistant sur un rôle spécifique, légalement reconnu. L'infirmière travaille toujours sous la responsabilité du médecin lorsqu'il lui prescrit les actes à accomplir mais elle est autonome lorsqu'elle agit selon son "rôle propre ${ }^{9}$. Celui-ci s'incarne à travers différentes actions notamment en matière de prévention et d'éducation à la santé. Si les infirmières ont agi au cours de leur histoire pour affirmer leurs compétences professionnelles et rompre avec l'image de la religieuse dévouée et soumise au savoir médical, elles paraissent toutefois avoir des difficultés à "tenir ensemble deux rôles que l'imaginaire oppose: le rôle propre et l'exécution d'actes prescrits. ${ }^{10}$ " Cela se traduit comme l'a expliqué E. Rothier-Bautzer ${ }^{11}$ par une inutilisation d'outils mis à leur disposition. L'auteure montre à travers le questionnement de l'utilisation des dossiers de soins, que les infirmières ne remplissent pas certaines cases concernant leur rôle propre, à savoir notamment les relations au patient et à sa famille. Mais cela ne veut pas dire qu'elles négligent pour autant les soins relationnels, fondateurs de leur identité professionnelle. Certes, elles valorisent la technicisation croissante de leurs actes, mais elles expriment cette double compétence, technique et relationnelle, à travers une approche globale des soins plutôt qu'en se référant à leur rôle propre ${ }^{12}$.

"Mon travail ? C'est un travail de soignant, on est là pour soigner les enfants en
prenant en compte différentes facettes. Il y a le soin en lui-même... il y a les
parents, donc il faut vachement de relationnel avec les parents, avec les enfants,
en prenant en compte leurs habitudes de vie, après il y a tout ce qui est
technique, l'hygiène tout ça. Après, la puériculture c'est un travail d'équipe avec
les autres puéricultrices, en binôme avec l'auxiliaire, avec les psychologues, les
médecins, les éducatrices et tout ça pour prendre l'enfant en charge de façon
globale... au niveau éducatif, relationnel, médical et psychologique. "
(Puéricultrice)

L'auxiliaire de puériculture est également un maillon de cette prise en charge globale de l'enfant, notamment par le "soutien à la parentalité ${ }^{13}$. Elle assiste l'infirmière lors des soins techniques mais il lui est formellement interdit de préparer et d'administrer un médicament. Comme elle est moins sollicitée que l'infirmière sur l'aspect purement technique du soin, l'auxiliaire de puériculture investit une part importante de son activité

\footnotetext{
${ }^{9}$ On retrouvera une liste des actes que l'infirmière peut accomplir dans le cadre de son " rôle propre » à l'article R4311-5, R4311-5-1 et R4311-6 du Code de la santé publique.

${ }^{10}$ Françoise ACKER, « La fonction de l'infirmière. L'imaginaire nécessaire », Sciences sociales et santé, vol 9, $n^{\circ} 2$, p. 123-143, 1991, p 136.

11 Eliane ROTHIER-BAUTZER, « Pratiques soignantes en mutation: de la lutte contre la maladie à la collaboration avec le patient ", Revue française de pédagogie, vol 138, p 39-50, 2002.

${ }^{12}$ France LERT, "Le rôle propre peut-il fonder l'autonomie professionnelle des infirmière ? ", Sciences sociales et santé, Vol 14, n³, 1996.

${ }^{13}$ Arrêté du 16 janvier 2006 relatif à la formation conduisant au diplôme d’Etat d'auxiliaire de puériculture.
} 
dans les soins d'hygiène et de confort, elle distribue les repas aux patients et stérilise le matériel. Elle réalise par là ce qu'A.M Arborio ${ }^{14}$ a identifié comme un travail invisible chez les aides-soignantes dans les services pour adultes. Ce travail est invisible car il est peu formalisé par des règles et souvent envisagé comme le sale boulot par les autres professionnels.

Il est cependant valorisé dans le discours des auxiliaires de puériculture comme gage d'une proximité avec le patient et sa famille. D'autant que l'infirmière perd ce contact au fur et à mesure que ses actes se technicisent et a alors tendance à déléguer à l'auxiliaire cet aspect du travail. Les auxiliaires jouent donc un grand rôle dans le relationnel avec les patients et leur famille surtout lorsque l'infirmière est affairée à la préparation des traitements. Plus longtemps au contact des usagers, elles se présentent souvent pour les autres professionnels comme pourvoyeuses d'informations utiles à la compréhension du comportement et de l'histoire de l'usager. En cela, sa proximité avec l'usager à travers le sale boulot constitue néanmoins une prérogative.

La collaboration étroite entre l'infirmière et l'auxiliaire se remarque dès le premier geste d'un soin technique, comme la pose d'une voie veineuse par exemple. Pendant que l'infirmière se concentre sur la manœuvre technique, l'auxiliaire se charge de "distraire " l'enfant. Alors que l'infirmière cherche la veine adéquate, l'auxiliaire l'assiste en préparant par exemple le MEOPA ${ }^{15}$ et le masque à appliquer sur le visage de l'enfant, occupe ce dernier soit en lui parlant, soit en lui chantant une chanson. Elle intervient, le cas échant, pour le tenir fermement afin que l'infirmière lui prodigue ses soins. Dans le service observé, le parent est généralement prié de ne pas assister aux prélèvements ou aux poses de voie car sa présence constitue, selon la rhétorique soignante, un facteur de stress pour l'enfant. "L'enfant ne comprend pas que le parent laisse le soignant lui faire du mal, donc il hurle encore plus et le réclame ". Aussi l'auxiliaire, si elle n'a pas un rôle direct à jouer dans l'acte technique, s'occupe de rassurer le parent resté en dehors de la salle de prélèvement.

\subsection{Le parent.}

II s'agit en réalité de parvenir à convaincre le parent de ne pas assister aux actes invasifs et douloureux. S'il insiste pour être présent, les soignants n'ont pas les moyens légaux de l'en empêcher. La question du droit à l'hôpital est essentielle car il est le levier qui a permis aux usagers de s'imposer comme décisionnaires dans la relation de service. A la suite de divers

\footnotetext{
${ }^{14}$ Anne-Marie ARBORIO, Un personnel invisible, les aides-soignantes à l'hôpital, Paris, Economica, 2002.

${ }^{15}$ Mélange gazeux analgésiant.
} 
épisodes judiciaires engagés par des malades à l'encontre de l'Hôpital ${ }^{16}$, ou à la suite de pressions exercées par les associations de malades, les usagers ont conduit l'Etat à reconnaitre leurs demandes et à les inscrire en tant que " coproducteurs " du service ${ }^{17}$. La loi du 4 mars 2002 ( $n^{\circ} 2002-303$ ) relative aux droits des malades et à la qualité du système de santé est emblématique de cette reconnaissance de l'usager en tant que "véritable acteur de santé $[\ldots]$ pour une médecine plus humaine ${ }^{18} »$.

Développer une " médecine plus humaine », plus démocratique, passe par un rééquilibrage des pouvoirs entre les soignants et les usagers et donc une transparence quant à l'activité des professionnels. En effet, des associations agréées sont autorisées à siéger au sein $d^{\prime}$ 'instances hospitalières, le malade peut consulter son dossier médical et il doit être informé de tout acte dont il est l'objet; bref l'usager à voix au chapitre. Si l'on reprend la thèse de F. Dubet $^{19}$ on comprend que l'usager, placé au cœur des dispositifs du nouveau management public, participe au déclin des programmes institutionnels en questionnant la légitimité et l'autorité de l'institution.

Concrètement, au sein du service observé, la coproduction prend alors la forme d'une rencontre de deux logiques souvent dissemblables mais pas toujours antinomiques. Les professionnels sont amenés à devoir composer avec les logiques familiales qu'ils rencontrent. Ils ne peuvent se permettre de les ignorer au risque que l'usager mécontent fasse appel aux appareils institutionnels qui lui ont reconnu cette nouvelle place. Néanmoins, il serait illusoire de croire que les soignants doivent tout mettre en œuvre pour contenter un usager-roi et qu'ils seraient totalement soumis à ses exigences. Ils disposent de marge de manœuvre pour contraindre l'usager car ils détiennent notamment les connaissances techniques nécessaires à l'exécution du service. En cela, ils sont souvent dominants dans la relation car ils savent ce qu'il faut faire et comment il faut le faire, à la différence de l'usager qui, en raison de sa méconnaissance des techniques, est dépendant du savoir des professionnels. Toutefois, l'usager détient un pouvoir de nuisance important, car conscient de ses droits, il peut passer rapidement de l'usager-consommateur à l'usagerplaignant $^{20}$. Aussi, cela participerait à l'appréciation subjective de l'augmentation des violences à l'hôpital comme en témoigne cette auxiliaire de puériculture.

\footnotetext{
${ }^{16}$ Laure AMAR, Etienne MINVIELLE, « L'action publique en faveur de l'usager : de la dynamique institutionnelle aux pratiques quotidiennes de travail » Le cas de l'obligation d'informer le malade, Sociologie du travail, vol 42, $n^{\circ} 1, p$ 69-89, 2000.

17 Jean-Marc WELLER, «La modernisation des services publics par l'usager : une revue de littérature (19861996) ", Sociologie du travail, Vol 40, n³, p 365-392, 1998.

${ }^{18}$ Elisabeth GUIGOU, "Projet de loi relatif aux droits des malades et à la qualité du système de santé ", Assemblée nationale, $\mathrm{n}^{\circ} 3258,127$ p, 2001, p 4-5.

${ }^{19}$ François DUBET, Le déclin de l'institution, Paris, Editions du Seuil, 2002.

20 Jacques DUBIN, « La place du patient », Esprit, n¹, p 40-51, 2007.
} 
"Depuis 25 ans que je suis à l'hosto, je peux dire que la violence a augmenté. Je suis régulièrement agressée par les parents qui sont en crise. Les chambres sont également un motif à conflit parce que la plupart des parents veulent avoir des chambres individuelles pour leurs enfants alors qu'on les réserve aux situations délicates du service comme les tumeurs ou les réanimations. [...] Les insultes on en a tous les jours. Tu essaies de les remettre à leur place. Tu leur montres que t'es là pour soigner, pas pour faire le gendarme. Il n'y a pas vraiment de règles, on fait au cas par cas, c'est-à-dire qu'on essaie toujours de faire retomber la tension. On parle doucement. Déjà tu leurs dis "bonjour ", ça met une barrière face à quelqu'un qui t'agresse directement. »

Travailler au contact d'un public implique de réaliser un travail émotionnel ${ }^{21}$, c'est-à-dire faire un travail sur ses propres émotions afin de les moduler et les adapter à la situation ou aux attentes émotionnelles de l'usager. Face à un parent angoissé par le diagnostic de son enfant, les soignants maîtrisent leur expression émotionnelle. Même confrontés à un diagnostic bénin, comme une infection urinaire par exemple, ils peuvent certes utiliser l'humour pour dédramatiser et rassurer le parent mais son usage est nécessairement limité par la réaction de ce dernier. Le travail émotionnel au sein du service n'est cependant pas soumis à un script établi des émotions à exprimer et de phrases-types à formuler auprès de l'usager, comme c'est le cas dans les centres d'appels ${ }^{22}$. En revanche, la prescription émotionnelle est bien présente comme l'illustre ce "recadrage » effectué par la cadre auprès de deux soignants se querellant au sujet du planning, en utilisant comme argument que "les parents ont des oreilles. Il faut essayer d'être calme parce qu'une ambiance électrique se fait sentir sur le soin. Les enfants, ils le ressentent le stress !» Ce rappel à l'ordre est révélateur de l'aspect collectif du travail émotionnel, lui-même constitutif du travail relationnel.

\subsection{Le travail relationnel.}

Si une règle prédomine au sein de l'équipe soignante, c'est bien l'impérieuse nécessité de créer un climat émotionnel serein pour éviter de troubler les enfants et les parents. Ce «travail sur les sentiments" de l'usager est indispensable à l'efficience du travail médical ${ }^{23}$. Un climat émotionnel propice au bon déroulement des soins est corrélé, pour les soignants, au degré de confiance que leur accordent les usagers. II apparait alors comme

\footnotetext{
${ }^{21}$ Arlie HOCHSCHILD, "Travail émotionnel, règles de sentiments et structure sociale », Travailler, vol 1, $\mathrm{n}^{\circ} 9, \mathrm{p}$. 19-49, 2003.

${ }^{22}$ Valérie GROSJEAN, Corinne RIBERT-VAN DE WEERDT, « Vers une psychologie ergonomique du bien-être et des émotions: les effets du contrôle dans les centres d’appels ", Le travail humain, vol 68, n4, p 355-378, 2005.

${ }^{23}$ Anselm STRAUSS, Shizuko FAGERAUGH, Barbara SUCZEK, Carolyn WIENER, "Sentimental work in the technologized hospital ", Sociology of Health and Illness, vol 4, n³, p 254-278, p 274, 1982.
} 
primordial d'instaurer une relation de confiance avec l'enfant et sa famille dès le début de I'hospitalisation. On peut voir les soignants bâtir cette relation de confiance aussitôt l'admission dans le service de l'enfant, lors du recueil de données ${ }^{24}$. L'infirmière qui le réalise cherche des informations utiles pour déterminer les actions à mener, c'est donc elle qui interroge l'usager. Mais elle prend aussi le temps de répondre aux questions des parents pour tenter de réduire leurs incertitudes. C'est justement la capacité des soignants à adopter une attitude attendue par l'usager et à délivrer des informations utiles pour lui qui est créateur de confiance ${ }^{25}$. Dès lors, la capacité de l'infirmière à apparaitre lors de cette première rencontre comme un personnel « ressource ", c'est-à-dire informé et empathique, est déterminante dans l'établissement de la relation de confiance. Cela renforce certes l'image d'un soignant fiable chez l'usager mais cela contribue aussi à ce que le soignant s'implique lui-même dans cette relation de confiance, comme le déclare cette puéricultrice.

"Avec les parents je me sentais beaucoup plus en difficulté au début. Mais comme ça fait deux ans et demi que je suis là, je connais mieux les pathologies, j'ai plus d'éléments de réponse pour les parents, je sais mieux comment les hospitalisations se passent. Je pense que le fait d'avoir plus de connaissances me permet d'être plus à l'aise avec les parents et de mieux répondre à leurs questions. Et du coup, cela me permet de plus pousser le relationnel avec les parents sans avoir peur qu'ils me posent une question et que je ne sache pas y répondre. »

Par ailleurs, on peut observer des stratégies de maintien de la confiance avec l'usager. L'une d'entre elle consiste à verbaliser systématiquement l'action que le professionnel réalise et à détailler oralement le processus de travail afin d'impliquer l'usager. Cela suppose de faire preuve de franchise comme quand une infirmière dit à un enfant que lorsqu'elle le piquera, il aura sans doute un peu mal. Prendre le risque de mentir aux usagers sur le travail effectué peut briser le travail relationnel accompli, puisque " la confiance se « donne »; on ne peut exiger qu'on nous l'accorde comme nous exigeons de ne pas être déçus, une fois qu'elle a été accordée ${ }^{26}$. Un des problèmes qui se pose alors c'est que l'usager est confronté à une multiplicité d'interlocuteurs. La confiance interpersonnelle qui lie le professionnel et l'usager est à chaque fois à reconstruire ${ }^{27}$. Même si l'on peut raisonnablement supposer que l'usager

\footnotetext{
${ }^{24}$ Le recueil de données vise à obtenir des parents, ou de l'enfant suivant son âge, des informations relatives à la maladie (objet de l'hospitalisation), à l'expérience de la pathologie (degré de connaissance sur la maladie), à des allergies éventuelles, à des habitudes de vie, à la situation maritale et professionnelle des parents, etc.

${ }^{25}$ Dominique PHANUEL, "Confiance dans les soins et soin de la confiance : la réponse relationnelle ", Politiques et management public, vol 20, $n^{\circ} 4, \mathrm{p} 115-132,2002$.

${ }^{26}$ Georg, SIMMEL, Sociologie. Etudes sur les formes de la socialisation, Paris, PUF, 1999, p 382.

${ }^{27}$ Michèle GROSJEAN, Michèle LACOSTE, Communication et intelligence collective. Le travail à I'hôpital, Paris, PUF, 2010.
} 
accorde un degré de confiance générale aux représentants de l'institution à laquelle il a affaire, on constate néanmoins que les professionnels personnalisent et individualisent la relation avec l'usager. Ils cherchent notamment à assurer une continuité dans l'interaction en restant sur le même secteur plusieurs jours d'affilés car : « Les patients te connaissent, ils te font confiance, il y a des liens qui se font. C'est bien qu'ils voient les mêmes têtes " (infirmière). Ils économisent ainsi du temps sur la recherche $d^{\prime}$ informations liées aux soins tout en évitant aussi d'avoir à reconstruire une relation de confiance à chaque fois.

En somme, le travail relationnel est produit, d'un point de vue pratique, pour apaiser l'usager éprouvé, inhiber son pouvoir de nuisance et donc cultiver un confort de travail. Mais le travail relationnel est aussi réalisé car il résulte de l'éthos professionnel des soignants. En effet, il est l'application concrète de l'idéologie du care. Cette doctrine du souci de l'autre ${ }^{28}$ est un moyen pour la mise en pratique de l'approche globale du soin qui se définit ellemême dans le fait de considérer l'ensemble des dimensions de l'individu et de pourvoir, ou tout du moins respecter son autonomie. Le care qui est à la fois pratiques et dispositions ${ }^{29}$, commande alors aux soignants d'impliquer le parent dans le processus de soin. Cela explique la présence d'une figure sociale $d u$ "bon » parent dans les représentations de l'équipe soignante qui s'inscrit dans leur logique professionnelle.

\section{2. le « bon " parent dans les représentations des soignantes.}

L'étude du discours soignant montre que le parent n'est pas une figure homogène. On peut au moins en relever deux, celle du " bon " parent qui correspond aux attentes sociales des soignants et qui joue alors un rôle attendu et a contrario la figure sociale du parent " critiquable » qui paraît en discordance par rapport aux attentes de l'équipe soignante voire quelque fois « inadapté » au parcours de soin de son enfant.

Le parent est évalué continuellement et discrètement par les soignants au regard de son rapport aux soins et à l'hospitalisation de son enfant. Lors des relèves, l'équipe soignante rend compte explicitement de cette évaluation. Durant celles-ci sont évoqués les prescriptions des médecins, le comportement des enfants et le comportement des parents. Même si ce dernier est en général moins discuté que les prescriptions médicales et le comportement des enfants, on peut entendre les soignants parler des parents comme: "Maman fait, papa commande mais ne fait rien ". L'état émotionnel des parents est également décrit: "Elle est angoissée pour tout cette maman» ou encore : "La maman c'est : pas trop vite le matin et doucement le soir. Elle s'occupe de lui que quand elle en a envie ». Les réactions déplacées des parents sont aussi soulignées durant les relèves: "

\footnotetext{
${ }^{28}$ Alice LE GOFF, "Care, empathie et justice un essai de problématisation », Revue du MAUSS, $\mathrm{n}^{\circ} 32, \mathrm{vol}^{2, \mathrm{p}}$ 203-241, 2008.

${ }^{29}$ Joan TRONTO, “Du care”, Revue du MAUSS, n³2, vol 2, p 243-265, 2008.
} 
(Infirmière sortante) Il y a une maman infirmière hier qui nous a fait un cirque pas possible parce qu'elle se plaignait du cathéter placé à son enfant. // (Auxiliaire) Moi, quand mes filles ont été hospitalisées, je les ai pas soulées puisque justement je suis de la profession. // (Infirmière rentrante) Bah t'inquiète pas! Elle ne va pas me gonfler longtemps cette maman.

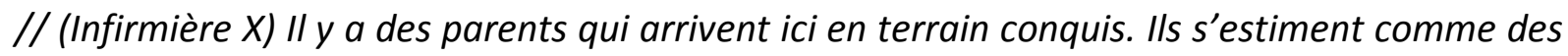
clients. On est leur personnel ». Mais on peut également les entendre parler positivement des parents, notamment des parents de patients chroniques qui excellent dans leur rôle. " $A$ la chambre 18, là c'est la mère qui s'occupe de tout. T'as rien à faire, tu la laisses faire, elle est mieux informée que nous cette maman. Elle s'occupe de sa gamine depuis si longtemps... "

Tous les parents ne sont pas mis en discussion au sein de l'équipe soignante car certains ne "posent pas de problèmes " ou non rien d'exceptionnel. En revanche, tous les parents sont soumis à une catégorisation sociale de la part des soignants en fonction principalement de leur rapport à l'hospitalisation de l'enfant et à sa pathologie. Cela constitue un critère important de la catégorisation sociale de l'usager qui s'opère alors en rapport à sa distance au rôle social du «bon » parent préétabli par les soignants. Cette figure sociale nous est explicitée par cette infirmière : "On a des familles qui ont confiance envers les soignants et qui s'en tiennent à ce que les médecins disent, sans demander plus et sans tomber dans la paranoïa ». Le "bon " parent apparaît alors comme celui qui a confiance dans l'action menée par l'équipe soignante et qui accepte le rôle proposé. Le "bon " parent s'oppose alors au parent "critiquable ", qui est défini comme " excessif " ou au contraire "pas du tout inquiété de la maladie de l'enfant " et ne comprend pas les soins donnés. Sont critiqués aussi les parents "passifs, qui amènent leur enfant à l'hôpital et une fois qu'on s'en est occupé viennent le chercher ". De même, les parents " tout le temps en opposition avec ce $q u$ 'on fait " sont également sujets à critiques de la part de l'équipe soignante. Par contraste, le « bon » parent apparaît comme celui qui s'adapte au fonctionnement de l'hôpital. Ce qui détermine l'action des soignants à l'égard du parent et donc sa catégorisation, c'est sa " compliance au traitement, l'adhésion à une norme d'autonomie vis-à-vis de l'hôpital [et] le respect des normes contemporaines à la parentalité ${ }^{30}$.

Cette figure du «bon» parent s'incarne assez souvent chez les parents de malades chroniques qui, fréquentant le milieu hospitalier depuis un certain temps ont intégré son fonctionnement, savent donner des soins à leur enfant, font preuve d'autonomie et connaissent la trajectoire médicale. Mais cela ne veut pas dire que tous les parents des patients chroniques sont considérés comme de «bons » parents. Beaucoup sont critiqués

\footnotetext{
${ }^{30}$ Sarra MOUGEL, "Introduire les parents à l'hôpital : enjeu thérapeutique ou prise en compte des émotions de l'enfant ", in Cédric LE BODIC et Anne-Chantal HARDY, (dir), Prescrire, proscrire. Enjeux non médicaux dans le champ de la santé, Rennes, Presses Universitaires de Rennes, p 205-218, p 218, 2012.
} 
justement par les soignants pour leur manque d'implication dans la pathologie alors même que l'aspect chronique de la maladie devrait les y pousser. Dans le service observé la question du diabète en est emblématique : "ll y a des parents et des ados qui pensent que le diabète ce n'est pas grave, qu'on peut ne pas surveiller sa glycémie. Ils ne se rendent pas compte qu'ils risquent de se foutrent les reins en l'air. Ça tiendrait qu'à moi, je ne les ferais pas partir de l'hôpital tant qu'ils n'ont pas pris conscience de ce qu'est la maladie, parce que s'ils partent sans comprendre la maladie, ils reviendront malheureusement très vite et en plus mauvais état " (infirmière). La figure du " bon " parent se trouve donc aussi parmi les familles de malades aigus, qui sont moins expérimentées et socialisées à la pathologie et aux logiques professionnelles. En d'autres termes, il n'apparaît pas que le " bon " parent soit uniquement celui qui "brille " par sa capacité à se substituer aux soignants, mais celui qui accorde sa logique d'action à celle de l'équipe soignante.

La catégorisation sociale est un moyen d'ordonner son environnement, de le simplifier, de lui donner du sens et d'exercer un contrôle sur lui ${ }^{31}$. De fait, il s'agit d'une réduction de la réalité sociale qui est parfois contestée par les soignants eux-mêmes. Effectivement, si l'on constate un relatif consensus quant aux critères qui distinguent un "bon " parent d'un parent "critiquable", les soignants indiquent également être parfois trompés par l'évaluation de leur collègue reposant sur des stéréotypes et réduisant alors l'usager à quelqu'une de ses caractéristiques. Dans tous les cas, si la catégorisation sociale de l'usager est quelque fois dénoncée par les soignants car perçue comme abusive, elle permet cependant d'ouvrir le débat sur ce qu'est le « vrai travail » et par là même de le définir, en délimitant notamment les actions à entreprendre et les limites de ces actions ${ }^{32}$.

\section{La place des parents, l'exemple de l'éducation thérapeutique.}

La figure sociale du « bon " parent dans les représentations de l'équipe soignante implique donc une catégorisation sociale de l'usager et une typification ${ }^{33}$ des situations de travail. Autrement dit, lorsque les soignants estiment qu'un parent ne relève pas de la catégorie des " bons " parents, cela signifie qu'il a des pratiques " inadaptées " aux soins de son enfant. Pour tenter de corriger ces pratiques et de construire alors un « bon » parent, les soignants mettent en œuvre des actions telle l'éducation thérapeutique.

Faire l'éducation thérapeutique des parents, c'est leur expliquer comment administrer un traitement à l'enfant. Cela consiste à apprendre des gestes techniques aux parents, ou à l'enfant quand il en a les capacités, pour qu'ils puissent les reproduire par la suite tous seuls,

\footnotetext{
${ }^{31}$ Véréna AEBISCHER, Dominique OBERLE, Le groupe en psychologie sociale, Paris, Dunod, 1998.

32 Valérie BOUSSARD, Marc LORIOL, Sandrine CAROLY, " Catégorisation des usagers et rhétorique professionnelle ». Le cas des policiers sur la voie publique, Sociologie du travail, n48, p 209-225, 2006.

${ }^{33}$ Peter BERGER, Thomas LUCKMANN, La construction sociale de la réalité, Paris, Armand Colin, 1996.
} 
en autonomie. Les infirmières enseignent par exemple aux parents de malades chroniques à réaliser une injection au valium lorsque leur enfant convulsera en dehors de l'hôpital suite à une crise d'épilepsie. Elles font également la démonstration de l'administration du traitement aux parents de malades aigus. Mais l'éducation peut dépasser le simple apprentissage d'un traitement et concerner l'hygiène de l'enfant, comme le montre cette observation.

«Dans la chambre du bébé secoué, l'auxiliaire s'adresse à la jeune mère : "Vous voyez, il a le nez encombré. Ça le gène et en plus il faut le nettoyer pour prévenir des rhumes. " Elle lui explique alors comment faire : " vous fixez l'embout sur le mouche-bébé, vous mettez du sérum physiologique dans le nez de votre enfant et vous aspirez grâce au mouche-bébé ". La maman paraît écœurée à l'idée de le faire, mais l'auxiliaire insiste " allez, il faut le faire ". Finalement la mère parvient à dégager le nez de son enfant, non sans grimaces et difficultés à le garder calme, l'auxiliaire la félicite "bravo, c'est très bien. " »

Cet extrait, en apparence superficiel puisqu'il ne s'agit que d'un nez obstrué, révèle pourtant des aspects importants de l'éducation thérapeutique. Après avoir expliqué à la mère la nécessité de nettoyer quotidiennement le nez de son enfant, en mobilisant un argumentaire médical, l'auxiliaire lui décrit le processus technique à mettre en œuvre. Elle incite la mère à être active (allez, il faut le faire), à s'engager à travers la réalisation du soin. In fine, elle félicite la mère pour l'encourager à reproduire ce geste. De plus, les parents de cet enfant font l'objet d'une "sévère " catégorisation sociale de la part de l'équipe soignante car les cas de maltraitance infantile sont aux antipodes de l'idéal professionnel des soignants. Et même si la responsabilité des parents dans les lésions neurologiques dont souffre le bébé n'était pas avérée (l'enquête de police était en cours), ils étaient cependant sujets à une surveillance étroite de la part de l'équipe concernant leur rapport à l'enfant, à travers la façon de s'en occuper, le prendre dans les bras, s'adresser à lui, etc., cela notamment pour déterminer les actions à entreprendre à leurs égards. En conséquence, l'éducation thérapeutique $n^{\prime}$ implique pas seulement d'apprendre des actes techniques aux parents. Cet apprentissage nécessite au préalable de sensibiliser les parents à la pathologie, en les rassurant ou en les alertant sur les risques liés à la maladie, il s'agit d'ajuster émotionnellement les parents à la situation de l'enfant.

L'apparente insouciance de certains parents quant à la maladie de leur enfant est discutée par les soignants, comme l'illustre cet extrait d'une relève : "Il y a un souci avec la maman de la chambre 11. Que la dialyse soit finie ou pas, elle l'arrête à $11 \mathrm{~h}$. Je lui ai dit de ne pas le faire, mais ça ne sert à rien. Elle ne comprend pas, alors j'accélère le processus pour que ça soit terminé à 11h. " L'infirmière indique trois éléments à ses collègues qui prendront le 
relais. Tout d'abord, elle signale le comportement inadapté de la mère envers le traitement dans le fait de l'interrompre. Ensuite, elle souligne sa tentative échouée de l'alerter et de lui faire comprendre l'intérêt de laisser la dialyse se poursuivre. Et même " si ça ne sert à rien ", ses collègues tenteront également de sensibiliser la mère. Enfin, l'infirmière justifie une stratégie mise en œuvre pour intégrer le comportement du parent, en accélérant le processus.

Si ces cas de figure sont contrariants dans le sens où les soignants voient leur action invalidée par les parents et doivent alors ruser pour prodiguer les soins, ils restent cependant marginaux en comparaison avec l'autre aspect de la sensibilisation qui vise à rassurer des parents angoissés. Pour les soignants, il est impératif que les parents et les enfants soient détendus afin de réduire les tensions qui peuvent perturber la mise en œuvre des actes techniques. "Les parents, ils sont agressifs parce qu'ils sont fatigués et stressés. II faut les rassurer » déclare une auxiliaire. Pour cela, rappelons-le, les soignants réalisent un travail sur les sentiments des parents afin de les apaiser et désamorcer des situations potentiellement conflictuelles et néfastes aux soins. Par le travail relationnel avec le parent qui implique donc à la fois un travail sur les sentiments de l'usager et un travail émotionnel sur les propres émotions des soignants, ceux-ci cherchent à normaliser le comportement attendu de la part des parents. Qu'ils acceptent notamment de s'éclipser de la salle de prélèvement alors même que l'enfant peut témoigner de vives émotions, mais qu'ils soient présents à la fin du prélèvement pour consoler l'enfant.

Ce travail relationnel avec le parent vise à l'ajuster émotionnellement mais aussi à l'installer dans un rôle à interpréter. Les soignants le familiarise au fonctionnement du service en fournissant des informations d'ordre pratique comme par exemple où se trouvent les biberons pour les bébés, quand utiliser la salle de bains collective, quand ils devront être disponibles pour la visite du médecin et quand ils pourront s'absenter du service en toute quiétude; bref des informations qui permettent au parent de s'inscrire dans la temporalité des soins. Elles sont certes données dès l'entrée dans le service de l'enfant lors du recueil de données, mais elles sont rappelées régulièrement lors des tours. La normalisation du rôle parental s'affirme encore plus nettement lorsque les soignants le socialisent à la pathologie en le formant au travail sur les sentiments de son enfant.

"Quand les gamins sont hospitalisés les parents ont tendance à tomber vraiment dans la maladie et à ne parler que de ça. Des fois on a des parents qui ne font que demander à leurs enfants " est-ce que tu as mal ? Est-ce que tu as mal ? Est-ce que tu as mal ? " Et ils oublient complètement que c'est un enfant de quatre ans qui aime jouer aussi. Du coup, ils n'amènent pas de jouets, ils ne leur proposent pas de lire des histoires et tout ça parce que "non! II a mal, ce n'est pas possible, 
il ne peut pas jouer ». Moi, j'essaie justement de rappeler aux parents que certes il y a l'hôpital, certes il y a la maladie, n'empêche, ça reste des enfants. On peut quand même essayer de faire un petit peu comme à la maison même si on est à l'hôpital. "

Dans cet exemple, l'infirmière en tentant de réajuster émotionnellement des parents obnubilés par la maladie de leur enfant, leur propose un rôle à interpréter. Cette demande soignante du rôle parental comme garant des habitudes de la maison est régulièrement rappelée durant l'hospitalisation. Lors du recueil de données, les soignants se renseignent sur les habitudes de vie de l'enfant en précisant au parent toute l'importance de ne pas rompre avec le style de vie de l'enfant à domicile malgré l'hospitalisation.

\section{Conclusion.}

L'équipe soignante participe à la consolidation du lien familial entre l'enfant et le parent, en formant ce dernier à jouer un rôle dans les soins de l'enfant. Pour apprendre au parent le rôle à interpréter, les soignants exercent un travail concret sur lui en le formant à certains actes techniques, en le sensibilisant à la pathologie, en lui expliquant ce qui est attendu de sa part durant l'hospitalisation. Pour mettre en œuvre ce travail, l'équipe procède au préalable à une activité de catégorisation sociale des usagers. Le parent, comme l'enfant, fait l'objet de discussions dès son entrée dans le service, il est soumis à un processus d'évaluation sociale et jaugé à l'aune de sa distance à un rôle prédéfini, institutionnalisé par l'équipe soignante. En d'autres termes, on constate que les soignants comparent le parent réel à une figure sociale "idéalisée " du rôle parental et que par un processus de socialisation secondaire, d'apprentissage des codes d'un nouveau milieu social, ils tentent de l'y conformer. Il s'agit de le normaliser par une régulation de contrôle.

Mais deux remarques s'imposent alors. Tout d'abord, nous l'avons vu dans le texte, les parents sont loin d'être une matière molle malléable à volonté. Certains n'acceptent pas le rôle proposé et contraignent l'équipe soignante à composer avec leur logique profane, leur régulation autonome. Alors que les soignants cherchent à encadrer et rendre actif le parent dans les soins selon leur idéal professionnel, ils se trouvent confrontés à des parents omniprésents et angoissés ou, à l'inverse, des parents absents et considérés comme apathiques dans les soins. Le paradigme des soins négociés permet alors de mettre en exergue les tensions, les compromis, les ruses employées, bref la régulation sociale mise en œuvre dans les interactions à l'hôpital. La deuxième remarque découle de la première. Si la construction sociale de la réalité dont témoigne la catégorisation sociale des usagers est effectivement un moyen pour "faire vivre l'idéal professionnel, faire comme si le " beau travail » pouvait être mené malgré le caractère plus prosaïque de l'expérience quotidienne 
et malgré les comportements plus erratiques des usagers en chair et en os. ${ }^{34}$, force est de constater que la tentative d'imposer l'idéal professionnel comme réalité objective se heurte à la subjectivité et l'hétérogénéité des usagers. Cela, les soignants en ont conscience lorsqu'ils déplorent en certaines occasions une catégorisation trop abusive et par conséquent trompeuse.

In fine, la normalisation du rôle parental n'est pas uniquement motivée par le dessein d'un parent se soumettant volontairement aux attentes des soignants. Certes, le travail relationnel peut être envisagé comme une stratégie visant à induire un comportement attendu chez l'usager et à le séduire pour inhiber son pouvoir de nuisance ; autrement dit, l'intégrer à la régulation de contrôle des soignants. Mais on ne peut négliger le fait que l'idéal professionnel, le soin global, s'articule autour du bien-être de l'enfant, de son rétablissement et de la construction $d u$ " bon " parent. En définitive, travailler sur le parent pour le modeler à l'image de l'idéal professionnel, en faire un «bon " parent, c'est une tentative de l'équipe soignante pour négocier l'accomplissement du «bon » travail et s'affirmer comme de « bons » soignants.

\footnotetext{
${ }^{34}$ Marc LORIOL, La construction du social. Souffrance, travail et catégorisation des usagers dans l'action publique, Rennes, Presses Universitaires de Rennes, 2012, p 163.
} 